\title{
Acta
Biochimica
}

Vol. 44 No. $2 / 1997$

$333-338$

QUARTERLY

\section{Specificity of the tonoplast transport of the oleanolic acid monoglycosides in the vacuoles from Calendula officinalis} leaves

\author{
Anna Szakiel and Wirginia Janiszowska \\ Institute of Biochemistry, Warsaw University, Al. F. Żwirki i S. Wigury 93, \\ 02-089 Warsaw, Poland
}

Received: 7 January, 1997; accepted: 20 April, 1997

Key words: Calendula officinalis, vacuoles, transport, oleanolic acid glycosides

The specificity of two separate tonoplast permeases transporting oleanolic acid glycosides was investigated in vacuoles isolated from leaf protoplasts of marigold (Calendula officinalis) with the use of chemically synthesized analogs. The results indicate that the proper structure of both parts of oleanolic acid monoglycoside, i.e. aglycon and the sugar moiety, are required for binding to a specific tonoplast carrier.

The vacuole in the plant cell can be regarded mainly as the storage compartment, important with respect to the accumulation of various compounds: inorganic salts, organic acids, sugars, amino acids, and secondary metabolites, including alkaloids, phenolics, terpenoids. Since most of these substances are synthesized in the cytoplasm, their efficient and selective transport through the tonoplast is obviously crucial for their storage inside the vacuole. Thus, much attention was paid to vacuolar transport systems in recent years. So far, several different mechanisms have been described for passage of various compounds across the tonoplast, i.e. simple or facilitated diffusion as well as energy-dependent carrier-mediated transport [1]. In turn, trapping mechanisms (such as ion-traps, binding to phenolic complexes or conformational changes) as well as ATPase or pyrophosphatase-generated protonantiport systems have been postulated as driving force for membrane passage and accumulation against a concentration gradient $[1,2]$.
Obviously, it is impossible to assume a general mechanism for the tonoplast transport of various natural plant products, because their structures and physicochemical properties differ dramatically. However, high specificity of the carrier-dependent transport of many compounds, including sugars, amino acids, organic acids, most alkaloids and flavonoid glycosides, is now doubtless [1-6]. Similarly, we have demonstrated that oleanolic acid glycosides, accumulating in significant amounts in Calendula officinalis leaf vacuoles, undergo translocation across the tonoplast by two different carrier-mediated processes, one of them being active, the other passive [7, 8]. Further results of competition experiments suggested that one carrier is common for all glucosides and the other for all glucuronides $[9,10]$. This is of great interest, because the glycosides belonging to each series differ from each other in the composition and length of sugar chain. On the other hand, some authors described extremely selective carriers, when even very small differences in the structure of the mole- 
cule, like the chirality at one carbon atom, could be decisive for the possibility of passing through the membrane [2].

Therefore, the aim of the present study was to investigate specificity of the oleanolic acid glycosides transport to the isolated vacuoles from Calendula officinalis leaf protoplasts.

\section{MATERIALS AND METHODS}

Isolation of protoplasts and vacuoles. Protoplasts were isolated from leaves of $C$. officinalis by macerozyme and cellulase lysis as described earlier [11]. Vacuoles were liberated-from protoplasts by polybase disruption with DEAE-dextran in isotonic conditions, then purified by centrifugation in discontinuous mannitol-sucrose-Ficoll gradient and afterwards stabilized as described previously $[8,12]$.

Precursors and analogs. Both monoglycosides and their methyl esters as well as tetraacetylglucoside and triacetylglucuronide were chemically synthesized with the use of either unlabelled or radioactive [3$\left.{ }^{3} \mathrm{H}\right]$ oleanolic acid as described earlier [13, 14]. The final specific activity of the obtained labelled compounds was $3.8 \mathrm{mCi} / \mathrm{mmol}$. The same method was used to obtain either unlabelled or radioactive mono- $\left[{ }^{14} \mathrm{C}\right]$ glucoside of cholesterol, with specific activity of 3.1 $\mathrm{mCi} / \mathrm{mmol}$.

Uptake experiments. The incubation procedures were carried out in standard conditions described previously, with or without $\operatorname{ATP}(1.5 \mathrm{mM})[7,8]$. Radioactive compounds $\left(9 \times 10^{4}\right.$ d.p.m. $/ 10^{5}$ vacuoles $)$ were administered in $1 \mathrm{ml}$ of standard incubation medium [8]. In competition experiments radioactive monoglycosides $\left(4.5 \times 10^{4}\right.$ d.p.m. $)$ were added to $10^{5}$ vacuoles simultaneously with an equal amount (5 nmoles) of unlabelled compound. The flavonoid aglycons and glycosides (1 $\mu$ mole) were administered to isolated vacuoles in the same conditions. The substances nonabsorbed to the vacuoles were washed off by centrifugation in the mannitol-sucrosegradient $[8,12]$.

Radioactivity measurements. The fractions of vacuoles purified after incubation were extracted with ethyl ether and n-bu- tanol [8]. Radioactivity of the compounds taken up was estimated in a Beckman scintillation counter.

\section{RESULTS AND DISCUSSION}

For a more precise characterization of the C. officinalis tonoplast transport system, we have investigated the uptake of various analogs of oleanolic acid monoglycosides (Fig. 1) by isolated vacuoles. The glycosides accumulating in the vacuoles are typically composed of an aglycon with a hydroxyl group and a sugar chain consisting of one or several sugar molecules. The aglycon, oleanolic acid, is a pentacyclic triterpenoid with one double bond and two functional groups: hydroxyl in $\mathrm{C}-3$ and carboxyl in C-17 position (Fig. 1);

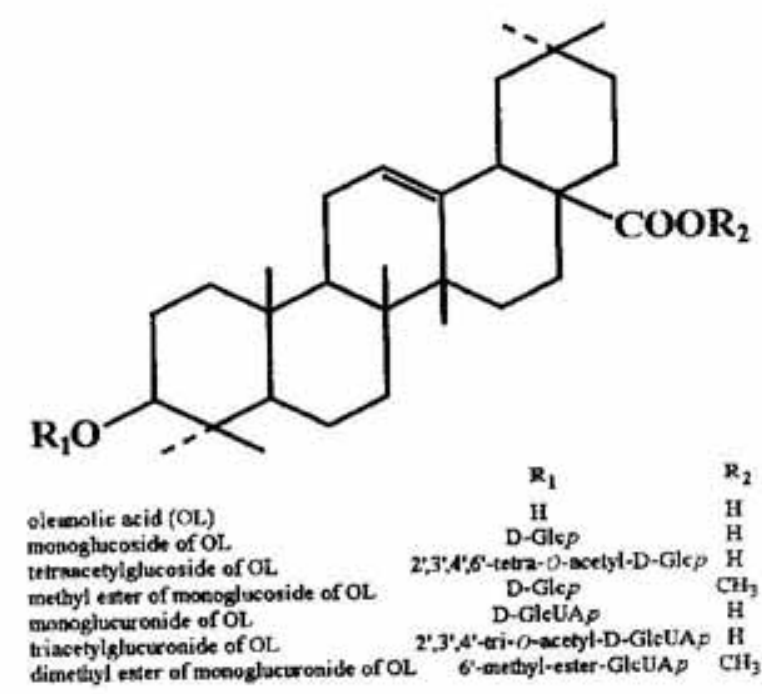

Figure 1. The structure of oleanolic acid, its monoglycosides and methyl and acetyl derivatives.

therefore, sugars can be attached to the aglycon molecule not only by a typical glycosidic linkage in $\mathrm{C}-3$, but also to carboxyl group in C-17. In turn, the carbohydrate component of oleanolic acid glycosides in $C$. officinalis is composed of glucose, galactose and glucuronic acid [15, 16]. Sugar chains of glycosides of the two series differ significantly in the first molecule attached to the aglycon (either glucose or glucuronic acid) as well as in the amount and sequence of sugars. Thus, it is of great interest which part of a transported molecule is decisive for its recognition 
by the respective carrier. The compounds tested were modified either in the sugar molecule (acetylated glucose, methylated or acetylated glucuronic acid) or in the aglycon (methylated oleanolic acid; cholesterol instead of oleanolic acid). The results are presented in Table 1 . Within experimental error, monoglucoside inhibited vacuolar uptake of radioactive monoglucoside in the absence (by $43 \%$ ) and in the presence (by $58 \%$ ) of ATP. The modified analogs of both oleanolic acid monoglucosides and cholesterol monoglucoside had no inhibitory effect on monoglucoside uptake. Obviously, they did not com-

Table 1. Specificity of monoglycoside transport to isolated vacuoles from Calendula officinalis.

The data were calculated as means of four independent experiments.

\begin{tabular}{lcc}
\hline Compound & Radioactivity incorporated (d.p.m./10 $0^{5}$ vacuoles) \\
\cline { 2 - 3 } & -ATP \\
\hline Monoglucoside of $\left[3-{ }^{3} \mathrm{H}\right]$ oleanolic acid & 25200 & 78600 \\
Methyl ester of $\left[3-{ }^{3} \mathrm{H}\right]$ oleanolic acid-monoglucoside & 25 & 28 \\
Tetracetylglucoside of $\left[3-{ }^{3} \mathrm{H}\right]$ oleanolic acid & 32 & 19 \\
Monoglucuronide of $\left[3-{ }^{3} \mathrm{H}\right]$ oleanolic acid & 22300 & 21900 \\
Dimethyl ester of $\left[3-{ }^{3} \mathrm{H}\right]$ oleanolic acid-monoglucuronide & 31 & 39 \\
Triacetylglucuronide of $\left[3-{ }^{3} \mathrm{H}\right]$ oleanolic acid & 22 & 25 \\
Mono- $\left[{ }^{14} \mathrm{C}\right]$ glucoside of cholesterol & 47 & 49
\end{tabular}

none of the analogs tested was found to be transported across the tonoplast, suggesting that the unchanged structure of the sugar moiety as well as the aglycon is probably prerequisite for their passing through the membrane. Apparently, the structural modifications of monoglycosides were too significant for the persistence of glycoside binding ability to the carrier. The data obtained indicate that the glycosides-transporting carriers of $C$. officinalis recognize not only differences in the amount and arrangement of rings and the presence of hydrocarbon chain in cholesterol instead to oleanolic acid, but also additional methyl or acetyl groups in the sugar moiety were found to be decisive.

The above conclusions were additionally confirmed by the results of the competition experiment presented in Table 2. Isolated vacuoles were incubated simultaneously with equal amounts of radioactive monoglycoside and its unlabelled analog. It was found that the tonoplast transport of radioactive oleanolic acid monoglycoside was affected only in the presence of the respective unlabelled compound. Thus, only unlabelled pete with oleanolic acid monoglucoside for the transport through the tonoplast. Likewise, the presence of oleanolic acid monoglucuronide exerted no effect on the transport of monoglucoside, as it was expected considering our earlier findings [9]. Similar results were obtained when the vacuolar uptake of oleanolic acid monoglucuronide was investigated. Again, none of the tested unlabelled analogs competed with the radioactive monoglucuronide in the tonoplast transport, whereas it was inhibited by unlabelled monoglucuronide by $60 \%$ in the absence, and by $45 \%$ in the presence of ATP. The presented findings strongly indicate that the binding sites of the carriers in vacuolar membrane must involve an extreme structural diversity and high sensitivity to modifications of transported molecules.

Our earlier results [8] of competition experiments with other glycosides of both series have indicated that each of the two carriers recognizes several related compounds, possessing the same aglycon and the first attached sugar (respectively, glucose or glucuronic acid linked to oleanolic acid) but dif- 
Table 2. Effect of various analogs on the transport of oleanolic acid (OL) monoglycosides to the isolated vacuoles from Calendula officinalis.

Competing unlabelled compound was added to the incubation medium at the same time and in the same amount as the radioactive monoglycoside. The presented values are means of at least three determinations.

\begin{tabular}{|c|c|c|c|c|c|}
\hline \multirow[t]{2}{*}{$\begin{array}{l}\text { Radioactive } \\
\text { monoglycoside }\end{array}$} & \multirow[t]{2}{*}{ Compound added } & \multicolumn{4}{|c|}{$\begin{array}{l}\text { Radioactivity incorporated } \\
\text { (d.p.m. } / 10^{5} \text { vacuoles) }\end{array}$} \\
\hline & & -ATP & $\%$ & +ATP & $\%$ \\
\hline $\begin{array}{l}\text { Monoglucoside of } \\
{\left[3-{ }^{3} \mathrm{H}\right]-\mathrm{OL}(10 \mathrm{nmole})}\end{array}$ & & 25200 & & 78600 & \\
\hline \multirow[t]{8}{*}{$\begin{array}{l}\text { Monoglucoside of } \\
{\left[3-{ }^{3} \mathrm{H}\right]-\mathrm{OL} \text { ( } 5 \text { nmole) }}\end{array}$} & & 12400 & 100 & 38100 & 100 \\
\hline & monoglucoside of OL & 7100 & 57 & 15900 & 42 \\
\hline & methyl ester of monoglucoside of $\mathrm{OL}$ & 12200 & 98 & 37800 & 99 \\
\hline & tetraacetylglucoside of OL & 12700 & 102 & 37900 & 99 \\
\hline & monoglucuronide of OL & 12600 & 101 & 37500 & 98 \\
\hline & dimethyl ester of monoglucuronide of $\mathrm{OL}$ & 12300 & 99 & 38300 & 101 \\
\hline & triacetyl-monoglucuronide of $\mathrm{OL}$ & 12500 & 101 & 37600 & 98 \\
\hline & glucoside of cholesterol & 12700 & 102 & 37800 & 99 \\
\hline $\begin{array}{l}\text { Monoglucuronide of } \\
{\left[3-{ }^{3} \mathrm{H}\right]-\mathrm{OL} \text { (10 nmole) }}\end{array}$ & & 22300 & & 21900 & \\
\hline \multirow[t]{8}{*}{$\begin{array}{l}\text { Monoglucuronide of } \\
{\left[3{ }^{3} \mathrm{H}\right]-\mathrm{OL} \text { ( } 5 \text { nmole) }}\end{array}$} & & 11600 & 100 & 11300 & 100 \\
\hline & monoglucuronide of $\mathrm{OL}$ & 4700 & 40 & 6200 & 55 \\
\hline & dimethyl ester of monoglucuronide of $\mathrm{OL}$ & 11800 & 101 & 11000 & 97 \\
\hline & triacetyl-monoglucuronide of $\mathrm{OL}$ & 10900 & 94 & 11400 & 101 \\
\hline & monoglucoside of $\mathrm{OL}$ & 11300 & 97 & 10500 & 93 \\
\hline & methyl ester of monoglucoside of OL & 10700 & 93 & 10800 & 96 \\
\hline & tetraacetyl-monoglucoside of $\mathrm{OL}$ & 11500 & 99 & 11100 & 98 \\
\hline & glucoside of cholesterol & 11800 & 101 & 10500 & 93 \\
\hline
\end{tabular}

fering significantly in composition and length of the rest of the sugar chain. Obviously, not the dimension but only certain characteristic features of the molecule are virtually crucial for successful binding to the permease.

Some authors described highly specific tonoplast carriers differing enormously in their selectivity. On the one hand, there are very selective permeases recognizing even very slight modifications in the molecule, such as chirality at one carbon atom [2] or cis-trans isomerism [6]. However, most carriers described so far display broader selectivity and they translocate a larger variety of structurally related compounds. For instance, the tonoplast permeases occur which are common for the whole group of di- and tricarbexylic acids $[1,5]$ or recognizing vari- 
Table 3. The transport of exogenous flavonoid aglycons and glyeosides and Neutral Red to the vacuoles isolated from Calendula officinalis.

The flavonoids and Neutral Red uptake was determined using spectrophotometric measurement of their vacuolar content at the indicated wavelength.

\begin{tabular}{lccc}
\hline \multirow{2}{*}{ Compound } & $\lambda_{\max }(\mathrm{nm})$ & $-\mathrm{c}$ Absorbance \\
\cline { 3 - 4 } & & 0.01 & + ATP \\
\hline Apiin & 333 & 0.01 & 0.00 \\
Apigenin & 336 & 0.02 & 0.01 \\
Quercetin & 374 & 0.00 & 0.01 \\
Quercitrin & 364 & 0.01 & 0.02 \\
Malvin & 534 & 0.02 & 0.01 \\
Malvidin & 542 & 0.02 & 0.00 \\
Peonin & 523 & 0.01 & 0.02 \\
Peonidin & 532 & 0.54 & 0.01 \\
Neutral Red & 550 & & 0.53 \\
\hline
\end{tabular}

ous carbohydrates with a terminal fructosyl residue [17].

It is often claimed that plant vacuoles preferentially take up endogenous compounds $[18,19]$. To verify this assumption, we have investigated the uptake by the isolated $C$. officinalis vacuoles of several exogenous flavonoids, their glycosides and synthetic basic dye - Neutral Red. The results are presented in Table 3 . Indeed, none of the flavonoid aglycons or glycosides was found to be taken up by the isolated vacuoles, contrary to Neutral Red which was readily transported and accumulated. The tonoplast transport of flavonoids was earlier investigated by some authors $[1,20]$ and it was shown to be carrier-mediated and specific, whereas Neutral Red is translocated across the tonoplast unspecifically by simple diffusion. Therefore, selectivity for endogenous compounds seems to be a typical property of the carrier-mediated tonoplast transport systems.

Considering the above presented findings we can recapitulate that the tonoplast transport system described for $C$. officinalis, involving two separate carriers (each common for several structurally related compounds) is another spectacular example of the occur- rance of specific permeases with broad selectivity. Such type of tonoplast transport seems to be a general phenomenon in plant vacuoles.

\section{REFERENCES}

1. Martinoia, E. (1992) Transport processes in vacuoles of higher plants. Bot. Acta 105, 232-245.

2. Deus-Neumann, B. \& Zenk, M.H. (1986) Accumulation of alkaloids in plant vacuoles does not involve an ion-trap mechanism. Planta 167, 44-53.

3. Getz, H.P., Thom, M. \& Maretzki, A. (1991) Proton and sucrose transport in isolated tonoplast vesicles from sugarcane stalk tissue. Physiol. Plant. 83, 404-410.

4. Thume, M. \& Dietz, K.J. (1991) Reconstitution of the tonoplast amino-acid carrier into liposomes. Planta 185, 569-575.

5. Martinoia, E., Vogt, E., Rentsch, D. \& Amrhein, N. (1991) Functional reconstitution of the malate carrier of barley mesophyll vacuoles in liposomes. Biochim. Biophys. Acta 1062, 271-278. 
6. Rataboul, P., Alibert, G., Boller, T. \& Boudet, A.M. (1985) Intracellular transport and vacuolar accumulation of o-coumaric acid glucoside in Melilotus alba protoplasts. Biochim. Biophys. Acta 816, 25-36.

7. Szakiel, A. \& Janiszowska, W. (1991) The effect of $\mathrm{pH}$ and ATP on the transport of oleanolic acid monoglycosides into isolated vacuoles of Calendula officinalis leaves. Acta Biochim. Polon. 38, 47-51.

8. Janiszowska, W. \& Szakiel, A. (1992) The transport of $\left[3-{ }^{3} \mathrm{H}\right]$-oleanolic acid and its monoglycosides to isolated vacuoles of protoplasts from Calendula officinalis leaves. Phy. tochemistry 31, 2993-2997.

9. Szakiel, A. \& Janiszowska, W. (1992) Competition between oleanolic acid glycosides in their transport to isolated vacuoles from Calendula officinalis leaf protoplasts. Acta Biochim. Polon, 39, 107-112.

10. Szakiel, A. \& Janiszowska, W. (1993) The kinetics of transport of oleanolic acid monoglycosides into vacuoles isolated from Calendula officinalis leaf protoplasts. Acta Biochim. Polon. 40, 136-138.

11. Auguścińska, E. \& Kasprzyk, Z. (1982) Studies on the labelling of triterpenoids in shoots and cells or protoplasts from Calendula officinalis leaves. Acta Biochim. Polon. 29, 7-15.

12. Szakiel, A. \& Kasprzyk, Z. (1989) Distribution of oleanolic acid glycosides in vacuoles and cell walls isolated from protoplasts and cells of Calendula officinalis leaves. Steroids 53, 501-511.
13. Janiszowska, W., Wiłkomirski, B. \& Kasprzyk, Z. (1980) Synthesis of oleanolic acid 3-O-monoglucoside. Pol. J. Chem. 54, 21472152.

14. Janiszowska, W., Wiłkomirski, B. \& Kasprzyk, Z. (1988) Synthesis of oleanolic acid glycosides. Pol. J. Chem. 62, 403-407.

15. Kasprzyk, Z. \& Wojciechowski, Z. (1967) The structure of triterpenic glycosides from the flowers of Calendula officinalis L. Phytochemistry 6, 69-75.

16. Wojciechowski, Z., Jelonkiewicz-Konador, A., Tomaszewski, M., Jankowski, J. \& Kasprzyk, Z. (1971) The structure of glucosides of oleanolic acid isolated from the roots of Calendula officinalis. Phytochemistry 10, 1121-1124.

17. Keller, F. (1991) Transport of stachyose and sucrose by vacuoles of Japanese artichoke (Stachys sieboldii) tubers. Plant Physiol. 98, 442-445.

18. Deus-Neuman, B. \& Zenk, M.H. (1984) A highly selective alkaloid uptake system in vacuoles of higher plants. Planta 162, 250260.

19. Ehmke, A., von Borstel, K. \& Hartman, T. (1988) Alkaloid $\mathrm{N}$-oxides as transport and vacuolar storage compounds of pyrrolizidine alkaloids in Senecio vulgaris L. Planta 176, 83-90.

20. Mattern, U., Reichenbach, C. \& Heller, W. (1986) Efficient uptake of flavonoids into parsley (Petroselinum hortense) vacuoles requires acylated glycosides. Planta 167, 183-189. 